\title{
AN EFFICIENT SKIN CANCER PROGNOSIS STRATEGY USING DEEP LEARNING TECHNIQUES
}

\author{
Dr. S. RANGA SWAMY \\ Associate Professor, Department of CSE, \\ Vignan's Institute of Management and Technology for Women, \\ Kondapur, Ghatkesar, Hyderabad, Telangana \\ E-Mail:sirisatiranga@gmail.com \\ Dr. C. SRINIVASA KUMAR \\ Professor, Department of CSE, \\ Vignan's Institute of Management and Technology for Women, \\ Kondapur, Ghatkesar, Hyderabad, Telangana \\ EMail:drcskumar41@gmail.com \\ Dr. A. Gauthami Latha \\ Professor, Department of CSE \\ Sridevi Women's Engineering College, \\ Vattinagulapally, Hyderabad. \\ 500075, Telangana, India \\ E-Mail: gauthamilatha@gmail.com
}

\begin{abstract}
To start the right treatment, identification of an indefinite skin lesion is necessary. Only highly trained dermatologists who can treat them with an early diagnosis and diagnose melanoma skin lesions. The classification of skin for melanoma Dermoscopic images is 70 percent. Due to the limited supply of expertise, systems that sort dermal growth as the autoimmune or metastatic tumor can serve as an early screening tool. This study provides a model of the Convolutional neural network trained for skin lesion images, from previously acquired features of the Highway Convolutional neural network (CNN). It does not require advanced preprocessing. In addition, the model not require much computing power to train. The Convolutional neural network (CNN) method achieves training accuracy of $50 \%$, and $70 \%$ of the test data have classification accuracy, low, moderate and high accuracy of the estimated damage.
\end{abstract}

Keywords: Classification taxonomy, Convolutional neural networks, Skin cancer, melanoma.

\section{Introduction}

Cancer is one of the primary reasons for death worldwide. Researchers and doctors face many challenges in tackling cancer. The American Cancer Society shows about one-lakh members had skin carcinoma. About 1.5 lakh members had pulmonary carcinoma, 0.5 lakh members had mammary gland carcinoma, and 30,000 members had prostate cancer. Seventeen thousand seven hundred and sixty people have died out of brain cancer in 2019 (American Cancer Society, New Cancer Release Report 2019) [3]. Many people saved because of diagnosing the carcinoma earlier. In general, visual inspection and manual methods used for this type of cancer can help with disease identification. This physical description of the therapeutic depiction is prolonged and has a high risk of errors. Computer-aided Design (CAD) systems were introduced in the early 1980s to develop therapeutic imaging [4]. Characteristic mining is an essential pace in acquiring artificial intelligence. [5-21] Various techniques for removing various kinds of carcinoma. However, there are drawbacks to these techniques, depending on characteristic mining. To secure the drawbacks and improve performance, see the illustration suggested in [22, 23]. An in-depth study has the advantage of directly producing high-quality raw images. In addition to an in-depth study, graphics processing units (GPUs) used parallel with feature processing and picture identification. For instance, neuromuscular neural networks can identify carcinoma [24].

Direct digital imaging is a popular method for medical diagnosis with new computing and device learning mechanisms. A variety of in-depth study molds developed and implemented within clinical identification because of their ability to discover designs in digital pictures. Convolutional Neural Network's best performance technology for image classification. Similarly, Convolutional Neural Network has made progress in several clinical picture analysis tasks, including classification and diagnosis. For example, the Convolutional Neural 
Network model utilized in categorizing metastatic and metastatic patches in man's dermatological cells Epiluminescence microscopic images.

Skin cancer is one of the leading factors for carcinoma in the United States (USA). Melanoma is a type of skin carcinoma that occurs due to unusual growth in the number of pigment-producing cells. In 2017, 87,110 new melanoma cases were diagnosed, according to the American Cancer Society, and approximately 9,730 people are likely to die from melanoma. Melanoma is twice as common as the rest of carcinomas. Apart from the United States worldwide, it is a community health risk. Identifying the disease earlier is a significant concern while handling an illness that has several therapies. These days, additional advanced tools with extremely skilled medical personnel are required to diagnose melanoma accurately and quickly. Epiluminescence microscopic is a unique method that gives superior quality pictures of skin, scheming light and filtering external skin reflections. However, suppose physicians not trained to diagnose melanoma from Epiluminescence microscopic images. In that case, the number of cases is at the wrong diagnosis.

Furthermore, the classification of specialist dermatologists for sorting melanoma is 70 percent. Hence, there is a significant demand for the automated image analysis systems in identifying melanoma from Epiluminescence microscopic images. Besides, it is challenging due to the size, texture, position, color, shape of the pictures along with the optical resemblance amid metastatic patches and non-metastatic patches. To overcome this challenge, some researchers suggest splitting up first. It tries to capture the area of the image with melanoma automatically. Melanoma is diagnosed based on the segmentation result. In [24] introduced a methodology for categorizing metastatic growth in which automatic threshold identification first made to differentiate patches from the underneath skin. The form, shade and design characteristics were summarized and applied to the optimization framework. The optical feature subsets determined for classification. However, with limited discretion, some methods may not work correctly.

New research efforts to improve computerized Epiluminescence microscopic picture examination to diagnose body outer cover disorders due to limited supply by dermatologists. Such a technique used as a diagnostic tool to define these images. Various computerized picture examination techniques utilized. Several operations utilize lower-grade visual image representation, separating algorithms, and scientific device learning methods like Convolutional Neural Network, KNN (K-Close Neighbors), and SVM (Support). Limited to Convolutional Neural Network has a collection of hard-wired modules. Every element classically has three modules; Bulky layers, uniting layers, thick layers. Convolutional Neural Network applies continuous separates to unprocessed statistics in order to collect low-top as well as high-end attributes, which used for classification in most applications. Convolutional Neural Network is excellent at detecting patterns in digital images because it is ideal for detecting melanoma from Epiluminescence microscopic images. Inspired by the recent activities of research groups that have used GPUs to train intensive Convolutional Neural Networks. The study aimed to enhance the selection of layers in the Convolutional Neural Network structure to understand the classification improved to distinguish high-level melanoma features from images. This research accomplished to find out how to achieve high accuracy and modeling in melanoma classification using Convolutional Neural Network. Recovery is the actual positive number divided by all the positives. Higher recall means less wrong. Positive. To categorize accurateness is the percentage of appropriately calculated cases in the picture data file. [20].

In the first component of this research work, specific modules (i), the latest module included multiple layers, (ii) training levels and learning rates, (iii) activation activities, and (iv) several Convolutional Neural Network structures with various improvers. The malignancy sorting presentation is equivalent to the entire loss and exactness of models skilled in the Convolutional Neural Network structure. Secondly, a Convolutional Neural Network model produced utilizing preferred hyperparameters from formerly tested structures to categorize skin patches for Epiluminescence microscopic descriptions with more exactness as well as sensitivity. Along with creating new convolutional Neural Network models, other frequently accessible CNN models also utilized. They are relevant to data files like Wisgenet, Alexnet Convolutional Neural Network and Google Inception V3 to evaluate a particular representation.

\section{Related work}

The artificial neural network (ANN) is a powerful technology compared to traditional statistical methods. They provide more accurate results than data regression models. ANN is a data processing structure distributed in parallel. It designed with connections to neurons (dendrites), each processing unit having a single output connection (exon). The information is entirely available at the internal memory of the processing unit. An important task of medical science is the accurate diagnosis of diseases. ANN is the most widely used and successful soft computing techniques for diagnosis. They play an essential role in taxonomy because of their ability to study and accumulate knowledge. ANN is compatible with the human brain, which contains neurons. Dendrites receive input signals from neurons around the neuron. These signals are processed in the neuronal cell 
body and then through the axon as output and output terminals to other neurons. Every year, the number of people affected is increasing, and malignancy has grabbed the attention of a large number of research efforts. Software solutions are to be improved to recognize and scrutinize Epiluminescence microscopic pictures of patients' skin patches.

The various outlooks are enormous. Every study includes various methods, such as low-level feature extracts and different machine learning methods. Various methods used in dermatology for the characterization of skin melanoma images. $\mathrm{ABCD}$ rule is a method used by dermatologists to classify melanoma, specifies asymmetry, border irregularity, Color Pattern and Diameter. In [3, 11], automated Dermatology tools for melanoma parameters based on $\mathrm{ABCD}$ rules and model identification protocols. Their study utilized three computerized algorithms for skin picture dispensing to identify suitable models for malignancy. The prototype detection system improved [4]. Achieved 90 percent accuracy and proven to be a reliable system for diagnosing melanoma. Study of [5] Effective CADX (computer-assisted diagnostics) developed to categorize melanocyte and non-melanocyte skin patches utilizing customary digital micrographs. Capturing traditional and new color features using SVM is in their style. The system achieved 89 and 91 percent sensitivity and specificity, respectively, and 90.64 accuracies. To assess Epiluminescence microscopic lesions, [6] report a method based on the ABCDE classification. E means artificial neural networks that reach development, image processing and 78 percent sensitivity and 90 percent specificity.

Input images include healthy and melanoma lesions. It confuses the nervous system's training because melanoma lesions are isolated from entire parts of the skin. However, as healthy skin grows, the main difference between healthy skin and discoloration disappears. The ulcer used to differentiate melanoma from the wrong condition. A significant component of melanoma detection is the separation of skin lesions from Epiluminescence microscopic images. Dermatologists to find the shape of skin lesions use it. Therefore, the classification of existing skin lesions follows three steps; i) Injury classification ii) Minted hybrid area and iii) Injury classification. Frequently, the predefining step $\mathrm{c}$ before dismantling in order to lessen the unwanted sound of the film. So many researchers implement segmentation in their image classification systems. Research work on handbooks and others. Introduced an automated taxonomy system that includes predefining, dissection random characteristic abstraction steps. A two-step classification applied to shrink the inaccuracy rate of malignancy cases. His proposed framework, which integrates $\mathrm{ABCD}$ rules and techniques for melanin-differentiation extraction, showed $100 \%$ exactness in the categorization of melanoma. In the journal, a study performed for sorting of malignancy based on categorization using SVM and Convolutional Neural Network. This kind of malignancy diagnosing system, as recommended by [7]. There are six steps in a row. The stages include color placement preprocessing, black frame, hairpin removal, wound-area separation, feature normalization, feature selection and classification. The SVM learning algorithm utilized for categorizing in this research work showed that about 90 percent understanding and 94 percent particularity attained.

Two strategies for classifying lesions based on the distinction between confined and wide-reaching distinctiveness of skin patches outlined [8]. This process implies binary classification for melanoma or heart disease, automatic segmentation, feature extraction and classification training. Others achieved 97 percent sensitivity and 82 percent specificity. Local methods for classifying melanoma skin lesions recently suggested. A program for categorizing lesions using the bag-of-feature approach (BOF) sketched. In their move toward, they symbolize each picture in the lesion $16 \times 16$ web. Using filters such as Gabor, they isolate 23 lesions in a dataset of dermatoscopy images, with 85 percent improved performance. In ImageNet 2012 Visual Recognition Challenge Classification Challenge, topping DNN (Deep Neural Networks) Computer Vision. In-depth learning techniques show excellent remedies for many operations, including natural language dispensation, verbal communication, facial detection, article recognition and picture classification $[13,14]$. There is a propensity to utilize in-depth learning types for more accurate therapeutic picture cataloging. Few study personnel has begun to use interactive neural networks (CNNs) to categorize malignancy because of its high-resolution potential. CNN is an in-depth learning method that applies filters that trained to extract particular features from complex systems into raw data automatically. In one research study, CNN classifiers trained in several trained clinical skin images. It reaches 82 percent sensitivity and 84 percent specificity to distinguish melanoma from empty cases. Cicero and others. Introduced a strategy to classify non-malignant melanosis lesions from melanoma. In his study, he categorized image datasets. To improve and normalize the image is replaced with a dataset rotation so that the deep network does not change.

The Deep Learning Framework Cafe and Deep Neural Network Reset as a character extraction tool utilized for assessment. A new method for detecting melanoma using the Complete Residual Network (FCRN) for exact skin patch using a newly planned multi-level related statistics project. In [10] detected melanoma in Epiluminescence microscopic images supported by the in-depth study, short coding and SVM learning algorithm. They claim that their method is useful because it uses readability study in the domain, transfers attribute from the domain of natural photographs and eliminates the need for detailed data. Wound separation and advanced preprocessing used for pure CNN's. To distinguish multi-stage symptoms from skin lesions to more accurately classify melanoma than sophisticated methods. Newer motion systems combine intensive learning with skillfully entrenched equipment 
learning methods to generate multiple ways of categorizing skin pictures. More accurate diagnosis of malignancy, this research work brought up a representation to make out malignancy positive Epiluminescence microscopic descriptions using the CNN model.

\section{Precise novel Convolutional Neural Network}

This section provides strategies to classify melanoma into malignant and benign for developing a Convolutional Neural Network model. With the help of the available dataset Convolutional Neural Network model is trained by skin lesion images. By choosing relevant features/parameters from different structures, we present some Novel Convolutional Neural Network models and structures with high accuracy. Model evaluation and its performance compared to some published network structures interactive Neural Network Architecture [12].
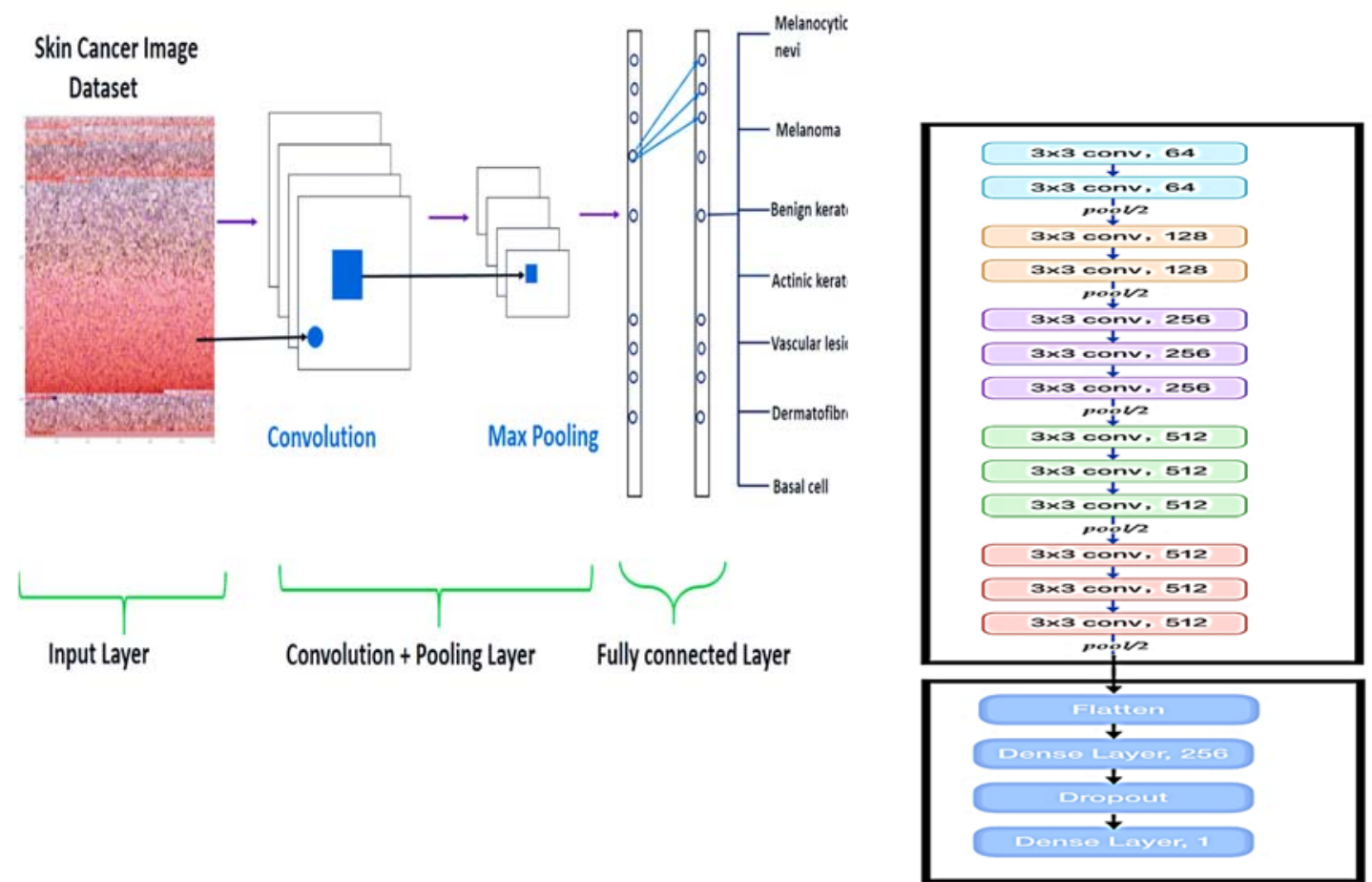

Figure-1: CNN layered approach for Cancer image dataset processing.

Figure-2: CNN layers for dataset processing.

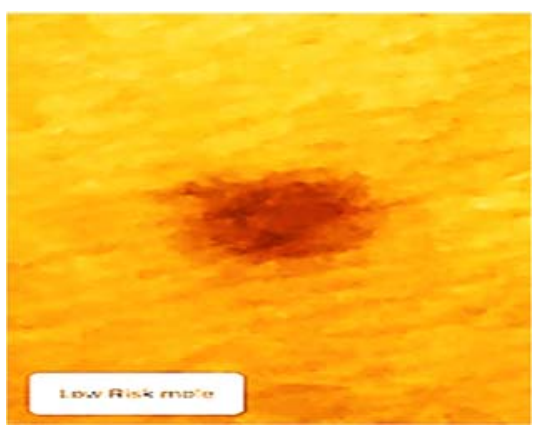

Figure 3: Skin low Risk Level

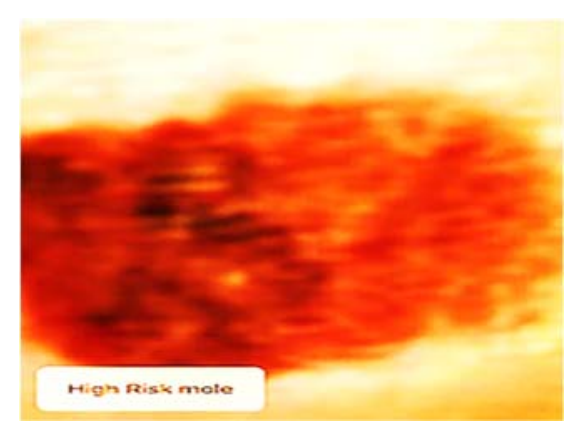

Figure 4: Skin high Risk Level

Various permanent neural networks Architectures assembled to categorize melanoma as malignant or empty.

1) Types of training stages and study rate.

2) Layers and their arrangement.

3) Adapter.

4) Stimulation function.

Detailed Convolutional Neural Network structures described in this section. To this network, some parameters added. The Convolutional Neural Network architecture comprises different layers. These include single and deep, 
fully adhered layers, pooling layers, and multiple activation functions. At all levels, a constant value maintained by the shape of the fixture kernel. It also tunes the number of filters in the corresponding layers. Later, Epiluminescence microscopic images created in various $\mathrm{CNN}$ architectures in the past. Figure 5 provides a characteristic diagram. For analysis of skin injury, Convolutional Neural Network architecture used. Categorize melanoma as malignant, four CNN structures developed before model development.

Algorithm Phase-1: Convolutional Neural Network Architecture1

1. Input: filter with $\mathrm{N}$ images, of mxm filter with for activation function (Constitution Layer).

2. Maintains maximum pooling with mxm filters step. (Pooling)

3. Activation function 64 filters with $2 \times 2$ relay (Neural layer).

4. Again, it maintains maximum combining and an advance of 1 with $2 \times 2$ filters Pooling layer.

5. Fully connected membrane: 2 neurons, one per class.

Algorithm Phase-2: Convolutional Neural Network Architecture2

1. Begin 32, mxm filter with relay function. (Constitution layer)

2. Maintains maximum pooling with mxm filters and one stride. (Pooling layer)

3. Begin 64 filters with $\mathrm{mxm}$ relay function. (Neural layer)

4. Maintains maximum pooling with $\mathrm{mxm}$ filters and one stride (Pooling layer).

5. Begin 32 filters $m x m$ with relay function (Neural layer).

6. Maintains maximum pooling with mxm filters and one stride. (Pooling layer)

7. Implements $64 \mathrm{mxm}$ filters relay activation function.( Neural Layer)

8. Again, it maintains maximum pooling and an advance of 1 with $\mathrm{mxm}$ filters. (Pooling Layer)

9. Fully connected membrane \# 1: 1024 exclusion rate of neurons 0.8 .

10. All membrane with m:n neurons assigned to each class.

Algorithm Phase-3: Convolutional Neural Network Architecture3

1. Phase-3 similar to phase-2 alike CNN Architecture

2. However, this structure has six narrow and six peak-pooling layers.

Algorithm Phase-4: Convolutional Neural Network Architecture4.

1. The architecture consists of ten optical layers and ten peak-pooling layers.

2. Same in Architecture 2 and 3.

The dataset contains 2236 Epilumine-scence microscopic images. Of these, some distributed for training and onevaluation and some selected as test data. A final addition of each Convolutional Neural Network architecture gives the class a chance according to the layer vote. At first, based on a total loss, an advanced Convolutional Neural Network structure was selected. Convolutional Neural Network architecture credited with creating the new CNN model with low losses and high accuracy. Three commonly used matrix accuracy (ACC), recall and attributes used to assess a particular system's performance. Describe the matrix as follows:

Sensitivity $($ Recall $)=$ TP $/$ TP + FN

Accuracy $=\mathrm{TP}+\mathrm{TN} / \mathrm{TP}+\mathrm{TN}+\mathrm{FP}+\mathrm{FN}$

Here, TP, TN, FP, and FN represents True positives, True Negatives, False Positives, and False Negatives.

\section{CNN Mathematical Model}

This proposal's main objective is to build a model capable of inferring the diagnosis of skin cancer, using the leastsquares method as an aid. We will use a machine learning technique called extreme machine learning (EML), which is detailed in section the details of ELM presented. To build a model capable of inferring a patient's diagnosis, we will use data collected from a biopsy to diagnose skin cancer performed previously. For this, we have a matrix Xtr $\varepsilon \mathrm{R}^{\mathrm{dxm}}$. The column vector $\mathrm{Y} X \operatorname{tr}(:, \mathrm{k}) \operatorname{tr} \in\{-$ contains 1,1$\} \mathrm{m}$, which data contains for the diagnosis value one if that of the patient. We have also been diagnosed with cancer and -1 if diagnosed with benign cancer. We can relate the matrix Xtr and the vector Ytr through a function:

$$
\mathrm{F}(\mathrm{X} \operatorname{tr}(:, \mathrm{k}))=\mathrm{Y} \operatorname{tr}(\mathrm{k}), \forall \mathrm{k}=1, \ldots, \mathrm{m}
$$

Based on data collected for each patient, we can infer which type of cancer they have. Note that it is challenging to find the exact $\mathrm{F}$ function. So to determine a function $\varphi$ that comes closest to $\mathrm{F}$ using the extreme learning 
machine technique, with the multilayer perceptron neural network and finding its $\alpha$ 's parameters using the minimum squares. The $\varphi$ function is defined as follows

$$
\Phi(x)=\alpha_{1} g_{1}(x)+\alpha_{1} g_{1}(x)+\ldots+\alpha_{n} g_{n}(x)=\sum_{i=1}^{n} \alpha_{i} g_{i}(x)
$$

where $\alpha 1, \alpha 2, \ldots, \alpha$ are parameters and the functions $\mathrm{g} 1, \mathrm{~g} 2, \ldots$, gn are given by

$$
g_{i}(x)=\tanh \left(\sum_{j-1}^{d} w_{i j *} x_{j}+b_{i}\right.
$$

where and we can write the function (1) in matrix terms:

$$
\Phi(x)=\alpha^{T} \tanh \left(W_{x}+b\right)
$$

Correspond that $\alpha=\left[\alpha 1, \alpha 2 \ldots, \alpha_{n}\right]^{\mathrm{T}}$ the wi and $\mathrm{Wb}=\mathrm{a}\left[\mathrm{b}_{1}, . . \mathrm{b}_{\mathrm{n}}\right]^{\mathrm{T}}$ is of dimension a vector in column. $\mathrm{x}$, whose We must line build the function $\varphi$. For this, to determine the matrix of the coefficients $\alpha$, and the matrix G, of dimension nxm, such that $G=\tanh \left(W_{x}+b\right)$, whose element $G(i, k)$ corresponds to the evaluation of the $i$-th function gi calculated in vector data of characteristics of the k-th patient. In an ELM, the vectors and scalar bi $\in$ $\mathrm{R}$ define the function gi generated randomly using a standard normal distribution.

\subsection{Least Squares Method:}

Having defined $\varphi(\mathrm{X} \operatorname{tr}(:, \mathrm{k})) \approx \mathrm{Y} \operatorname{tr}(\mathrm{k}), \forall \mathrm{k}=1, \ldots, \mathrm{m}$, and the functions $\mathrm{g}(\mathrm{i}) \forall \mathrm{i}=1, \ldots, \mathrm{n}$, we can find each $\alpha$ associated with $\mathrm{g}$ (i) by the method of least squares. Finding $\varphi(\mathrm{X} \operatorname{tr}(:, \mathrm{k}))$ that most closely matches $\mathrm{Ytr}(\mathrm{k})$ means that the sum of the squares of the deviations $(\varphi(X \operatorname{tr}(:, \mathrm{k}))-\mathrm{Y} \operatorname{tr}(\mathrm{k}))$ is minimal, that is, We know that the minimum of $J(\alpha 1, \ldots, \alpha$ ) occurs when: Note that the linear system can express the above expression.

$$
A \alpha=C
$$

Where $A=\left(a_{i j}\right) \in R_{n \times n}, \alpha=\left(\alpha_{j}\right) \in R_{n}$ and $C=\left(c_{i}\right) \in R_{n}$, with

$$
\begin{aligned}
& A_{i j}=\sum_{k=1}^{m}\left(g_{i}\left(X_{t r}(: k)\right) g_{j}(X \operatorname{tr}(: k))\right) \\
& \text { and } \sum_{k=1}^{m}\left(Y_{t r}(k) g_{i}\left(X_{t r}(: k)\right)\right)
\end{aligned}
$$

So, need to find matrices A and C as described above and solve the linear system to find the vector $\alpha$. That done, we can now rewrite equation (3) in matrix terms, such that:

$$
\begin{aligned}
& S_{k}=\varphi\left(X_{t r}\left(: k_{j}\right)\right) \\
& S_{k}=a^{T} G \quad--(7)
\end{aligned}
$$

Whether $S_{k}=\alpha^{T} G$ is the vector containing information about the biopsy of the k-th patient, the diagnosis made as follows: A decision threshold and $\mathrm{y}_{\mathrm{th}} \in\{-1,1\} \mathrm{m}$, where $\mathrm{h}$ is a natural number that identifies the threshold. We will initially use thresholds $\mathrm{L}=\{-2,0,2\}$. Therefore, we will have their values saved in the vectors yt1, yt2 and yt3, respectively. Therefore, we were able to get results in $\mathrm{m}$ and analyze the effectiveness of our model.Y $\mathrm{t}$ $\mathrm{h} \in\{-1,1\} \mathrm{m}$ to compare. With $\mathrm{Y}$ tr $\in\{-1,1\}$ The system's performance can be measured quantitatively, for example, by calculating the accuracy (AC) or the false-negative rate (TFN) defined respectively by the equations:

$$
\begin{aligned}
& \text { Accuracy }=\frac{(\text { Number of patients correctly diagnosed by the system })}{\text { (Total Number of patients) }} \\
& \text { TFN }=\frac{\text { Number of cancer patients diagnosed as benign by the system }}{\text { (Number of patients who have cancer) }}
\end{aligned}
$$


Interpret the threshold and comment on the accuracy values and the false-negative rate obtained in the previous item. By assigning the thresholds given in the previous item, we noticed that the lower the threshold, the lower the rate of false negatives, but the accuracy also decreases. With these observations, we must consider the choice of the threshold, making the rate of correct results high, but with low values for false negatives. These represent severe errors and irreversible damage to patients. It is interesting to indicate that the threshold is directly related to how conservative want to be. Higher thresholds represent greater accuracy values but the enormous scope of severe errors such as false negative. In contrast, lower threshold choices demonstrate conservative choices, since the hit rate is lower. However, not many mistakes made about the false-negative rate.

Table 1: False negative rate and Accuracy values for given thresholds

\begin{tabular}{|l|l|l|}
\hline Threshold & Negative False Rate & Accuracy \\
\hline 2 & 0.12329 & 0.93639 \\
\hline 0 & 0.047945 & 0.91094 \\
\hline-2 & 0.020548 & 0.87786 \\
\hline
\end{tabular}

Table-2: Accuracy values and false negative rate for threshold -4.5 training data

\begin{tabular}{|l|l|l|}
\hline Threshold & Negative False Rate & Accuracy \\
\hline-4.5 & 0.0068493 & 0.75827 \\
\hline
\end{tabular}

Table-3: Accuracy values and false negative rate for threshold -4.5 in test data.

\begin{tabular}{|l|l|l|}
\hline Threshold & Negative False Rate & Accuracy \\
\hline-4.5 & 0 & 0.77273 \\
\hline
\end{tabular}

A false negative can incur irreversible damage to the patient. It has cancer that was not detected by the system. Because of this, determine the best value for the decision threshold L, which ensures a false negative rate of less than $1 \%$. As seen in the previous question, the lower the threshold, the lower the false-negative rate. To have a rate of less than $1 \%$, we will take values less than -2 but still have significant assertion. Note that the false-negative rate is practically nil. However, we still maintain an accuracy above $70 \%$, which represents more conservative yet efficient results. Based on the threshold presented in item 4, we realized that the results of the test data are compatible with the results of the training data. We maintained a reasonable rate of correct answers in the diagnoses, making a few serious errors (false negatives). Note that besides, the results obtained in both cases are very close, showing that the model built is not biased and concludes us on the topic.

With the studies carried out through this proposal, we saw how using technology applied to mathematical concepts can help everyday situations such as a medical diagnosis. In particular, we saw that the multilayer perceptron neural network, associated with the least-squares resolution method, was efficient in helping to diagnose skin cancer. It is crucial to indicate that the design presented does not replace the evaluation of a specialist. Instead, it adds knowledge and makes the results more accurate and efficient.

\section{Results and discussion}

With early clinical screening, skin cancer is like the most common human heart disease, diagnosed primarily visually. Subsequently, dermoscopic analysis, biopsy and histopathological examination performed. Sudden classification of skin lesions using images is a challenging task, as for microscopic inflammation changes in the presence of skin lesions. Dataset of 10000 training images with HAM10000 anti-human machine. It contains 10015 dermatoscopic images released as training sets for educational machine learning purposes. It is generally available through the ISIC archive. This benchmark data used to compare machine learning and human skill. There are seven different skin cancer classes, including actinic keratosis, basal cell carcinoma, benign Keratosis, Dermatofibroma, Melanocyte Nevi, melanoma, and vascular lesions. 


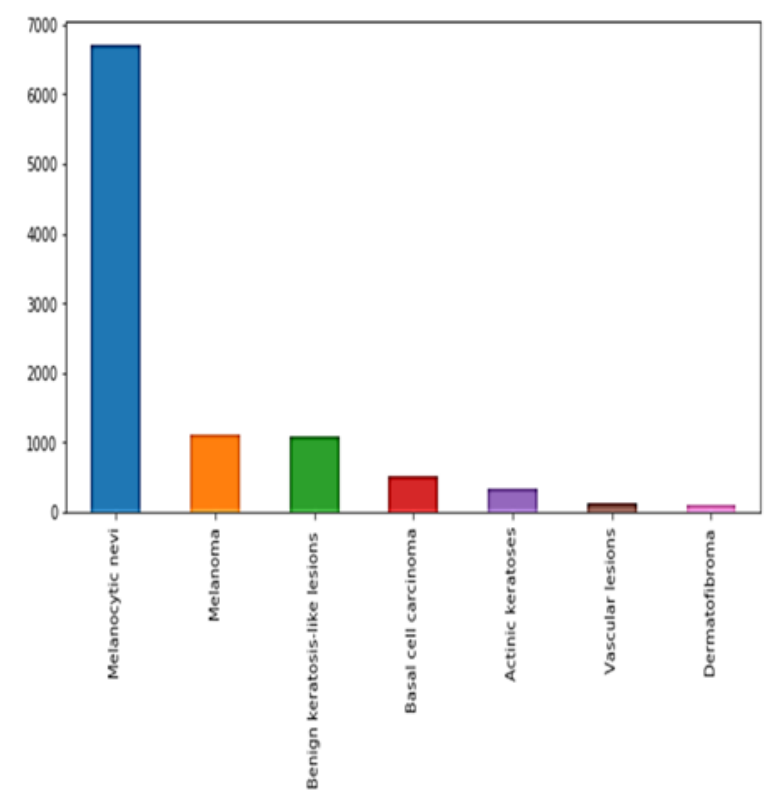

Figure-5: Different features of the dataset
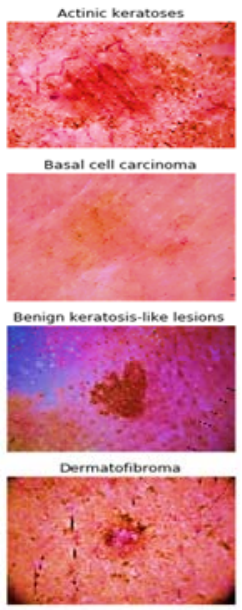

Melanocytic nev
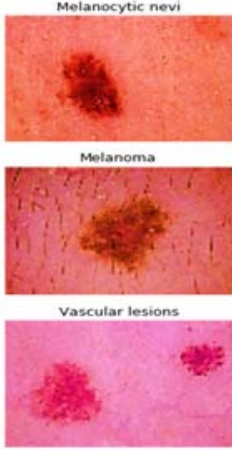
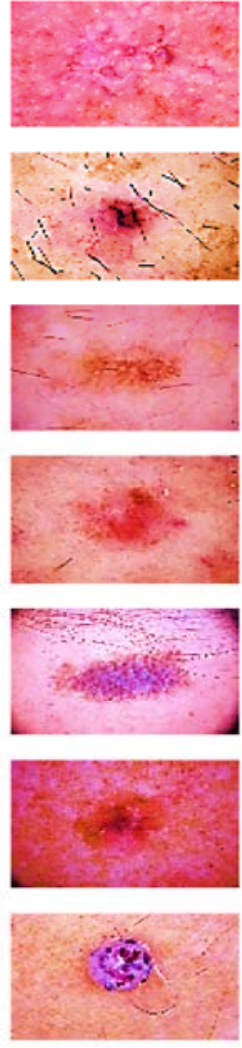

Figure 7: A sample of Skin Cancer images to identify cancer type.
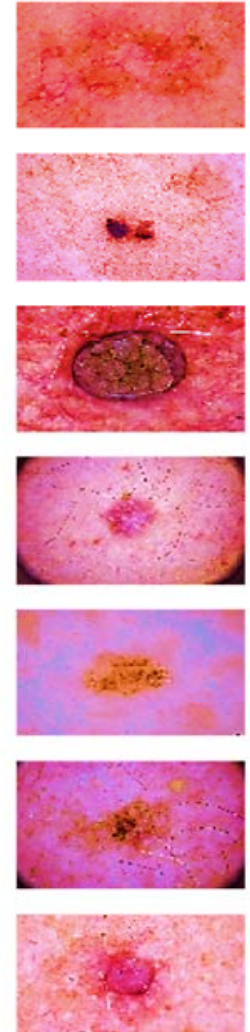

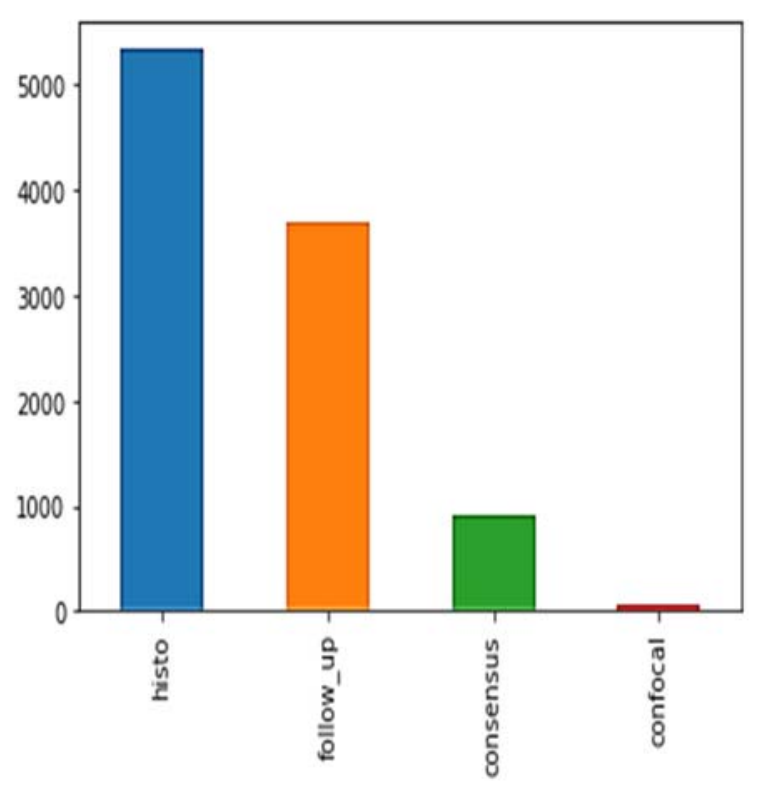

Figure-6: Feature spreading of localization field.
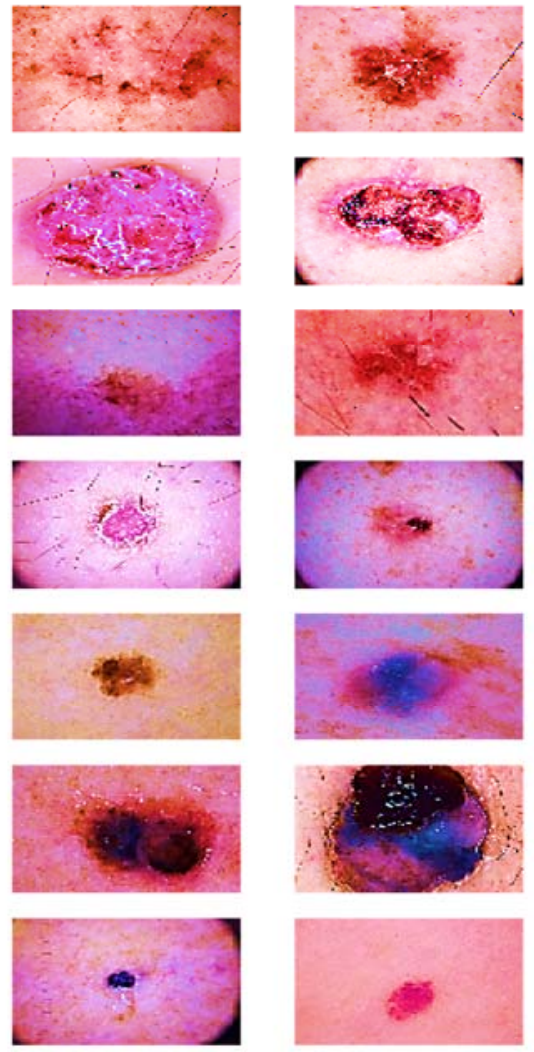

Types of skin cancers 0, 1, 3, 5 found in Melanocyte Navy, Dermatofibroma, basal cell carcinoma and vascular lesions under 20 years of age. At this point, the images loaded from the image folder to the image path. Since the actual dimensions of the images are $450 \times 600 \times 3$, we resize the images, which cannot handle the tensor flow. So let us change this to $100 \times 75$. This step changes the dimensions of all 10015 images to $100 \mathrm{x} 75$, so be patient. It may take some time. The most exciting part is that we show pictures of each cancer type always to look beautiful. The transaction layer is first, which is like a set of filters, simulating 32 filters for 2 first-cast 2D layers and 64 filters for 2 final casts. With the kernel, each filter replaces filter portion of the image. All over the image, the kernel filter matrix applied. Pooling (Max pool 2D) layer is the second most layer on Convolutional Neural Network, selecting the maximum value to reduce the calculation costs. This layer acts as a downsampling filter at 
two neighboring pixels, which are adequate. It chooses the pool size, each time the pool size increases, the area size and the pattern required.

By adding decorative and pooling layers, Convolutional Neural Network can study features of the image and add some features. To each training pattern, part of nodes randomly ignored in the layer. It randomly reduces the network ratio and prompts to learn how to distribute features. This method improves normalization and reduces over-fitting. Purification activation functions used to add lubrication to the network. The last layer is the flat layer. It requires the use of fully integrated layers after a few flat/maximum pool layers by which all the local properties of the previous concentration layers combined. Net output distribution of each class potential in the last layer (dense $(10$, activation $=$ "softmax")). After adding our layers to the model, we defined a loss function to find out how poorly our model works on badly labeled images and set the loss, score function and optimization algorithm. It is the error rate between the observed labels and the icon labels. Two classes, such as specific form $>$ hierarchy_crosscentrop, are used in a hierarchical configuration. The optimizer is the most important task which used to reduce damage by improving the parameters (kernel values, weight, a bias of neurons). Merits of the other two extensions of continuous origin, some optimism is noted here. Specifically: 1. Degrade (adaptive gradient algorithm) improves the performance of rare slope problems, language, computer vision problems and also sustains a learning rate for each parameter. 2. RMSProp (Root Mean Square Broadcast) - Based on recent volumes, RMSProp also sustains a learning rate for each parameter. Bulk gradients change rapidly, meaning the algorithm works well on online and non-consistent issues. Adam discovered the merits of Adam Grad and RMS Esprop. ADAM is a well-known algorithm in the field of in-depth learning that achieves excellent results quickly. Metric function accuracy used to estimate our model performance. This method is comparable to the loss function because when evaluating the model estimation result is not used. We used the Study Rate (LR) analysis method to make optimizer low loss operation worldwide. The phase moving through the optimizer' loss condition' is LR. More LRs takes more significant steps, faster integration. However, the higher LR model is shorter, and the adapter may stick to the local minimum. Decreased study during training is the best way to reach the overall global minimum loss. If necessary, we dynamically reduce the LRs of each X-phase age to take advantage of faster computing time with higher LRs. In the ischemic phase, the dataset expanded to ignore the problem. With minor changes, we use to reconstruct the differences for replacing the data. Data enhancement methods are ways to label training data and change the representation of an array. The most popular enhancements used by people are Grayscale, Horizontal Flip, Vertical Flip, Random Crops, Color Shifting, Translation and Rotation. Can generate more argument and increase training events by applying these two alterations to our data. We review the accuracy of our model test and the accuracy of citation evaluation during this time, resolve confusion, and examine each type of erroneous image. We can further improve our design to achieve accuracy higher than $80 \%$ accuracy. However, this model is useful compared to research, with $80 \%$ accuracy for the human eye. Our model seems to have the maximum number of false predictions for basal cell carcinoma, including code 3 . Vascular injury code five and Melanocyte Navy code 0 is the second unclassified type. Actinic keratosis code 4 is the lowest type of code.

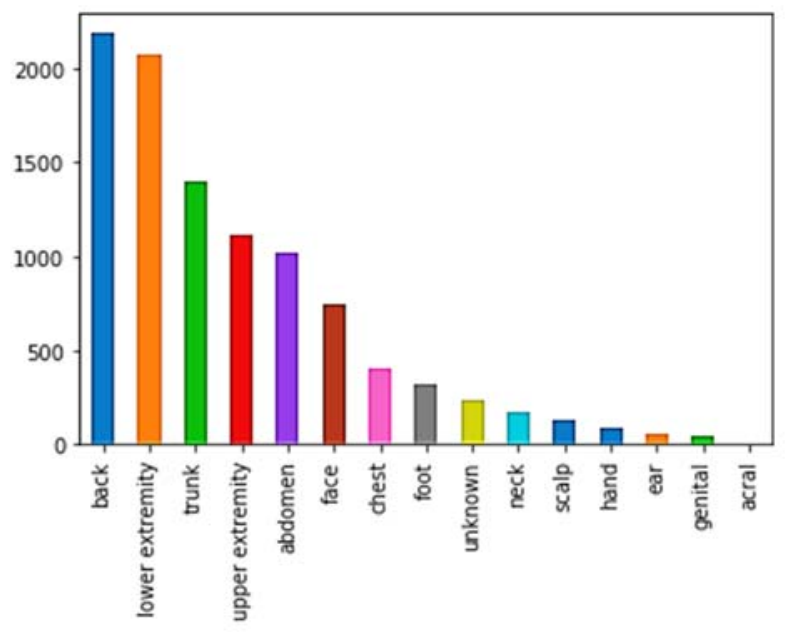

Figure-8: Different features of the dataset

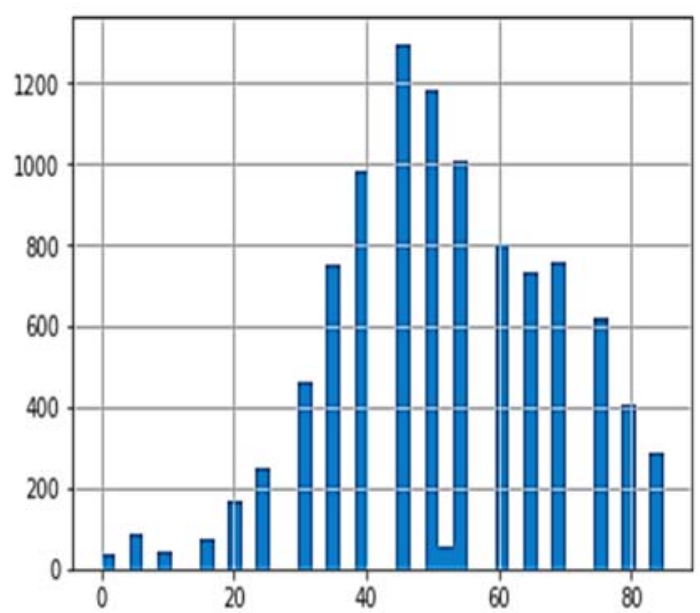

Figure-9: Feature spreading of localization field.

In this study, a new CNN model that optimizes CNN construction conditions based on built-in performance proposed. All CNN structures made with the tensor flow provides a high-level API to build the nervous system quickly. It facilitates thick layers, dense layer forms, a set of activation functions and simple dots. All CNN structures created in this study included multiple stable layers, layers fully integrated with pooling layers, and dropout layers. Thick layers the deflector filter is applied to the image. Single value on the output map produced 
by mathematical operations on each subfield in the map performs layer. Minimizing sub-layers can reduce the feature map size with some function. Maximum pooling is used in CNN architecture to capture parts of the feature map, maintain and discard maximum values. Complete layers are classified based on the properties extracted from the thick layers. The minimize additional fittings, the drop out layer is determined. In the development of CNN models for taxonomy, it usually refers to questions.

Nevertheless, no appropriate method to accomplish it. Therefore, to find the right combination choosing hyperparameters is one of the ways. A simple Convolutional Neural Network utility for extraction designed with layers. Presently, by altering the number of layers and their layout, four types of Convolutional Neural Network structures are created. The purpose of using different CNN structures is to identify the appropriate structure that can extract most melanoma symptoms from dermoscopic lesions.

In contrast, the other three structures have two interconnected layers. The second of these three structures is the failure layer, which used to pooling the layer. We can change two parameters to modify each layer's behavior by adding more layers in the Convolutional Neural Network structure. Stride and padding selected with the help of kernel size. The shape of the fixture layer, along with its input size and kernel size, affects zero paddings and stride selection. Kernel movement, kernel translation output handling controlled by stride. Volume Output the output volume is not an integer, but a fraction usually set to the stride. All CNN structures in this study set to Stride 1. If set to Stride 1, the receptive fields overlap, which increases the capture capacity of the feature. As indicated by the convection layers, it rapidly decreases the volume output. If the input maintained in the same way as the actual input volume, it would save many facts of source contribution capacity. The actual input volume is equal to the input-output volume by applying zero paddings. Therefore, zero-padding added to all hard layers of the generated $\mathrm{CNN}$ architecture.

\section{Conclusion}

This paper refers to the novel Convolutional Neural Network for classifying melanoma from dermatoscopic images of the skin. Evaluation of the method of detecting melanoma is available at the public level. According to $\mathrm{CNN}$, structural conditions this paper provides a comprehensive evaluation of samples. Furthermore, training to classify melanoma as benign and non-malignant using high evaluation correctness and little destruction. Different layers, activation functions, filters and their sizes, optimizers and fixed dot range are updated to tutor the model performance of this work. A similar data file used to tutor advanced network models like Alexander, Highway Convolutional Neural Network, Vigizenet and Google Inception V3. A new neural network model was advised based on each tested Convolutional Neural Network architecture and advanced network performance. About $97 \%$ of proper training and $68 \%$ certification accuracy, similar to cutting-edge networks by Deep Highway Convolutional with batch normalization of the proposed model.

\section{References:}

[1]. Jha, S., Mehta, A. K., \& Azad, C. (2020). A fuzzy logic-based approach for predicting basal cell carcinoma and squamous cell carcinoma is among the data of skin cancer. EAI Endorsed Trans. Pervasive Health Technol., 5(20), e4.

[2]. Lima, S., Terán, L., \&Portmann, E. (2020, April). A Proposal for an Explainable Fuzzy-based Deep Learning System for Skin Cancer Prediction. In 2020 Seventh International Conference on eDemocracy\&eGovernment (ICEDEG) (pp. 29-35). IEEE.

[3]. Coppola, D., Kuan Lee, H., \& Guan, C. (2020). Interpreting Mechanisms of Prediction for Skin Cancer Diagnosis Using Multi-Task Learning. Proceedings of the IEEE/CVF Conference on Computer Vision and Pattern Recognition Workshops (pp. 734-735).

[4]. Putra, T. A., Rufaida, S. I., \&Leu, J. S. (2020). Enhanced Skin Condition Prediction Through Machine Learning Using Dynamic Training and Testing Augmentation. IEEE Access, 8, 40536-40546.

[5]. Friedman, J., Hastie, T., \& Tibshirani, R. (2020). Discussion of "Prediction, Estimation, and Attribution" by Bradley Efron. Journal of the American Statistical Association, 115(530), 665-666.

[6]. Nair, N., Du, D., Hu, Z., \&Gongora, E. (2020). Risk Prediction Model for Basal Cell Carcinoma in Cardiac Allograft Recipients. The Journal of Heart and Lung Transplantation, 39(4), S128.

[7]. Wang, H. H., Liang, C. W., \& Li, Y. C. (2020). Application of Basic Epidemiologic Principles and Electronic Health Records in a Deep Learning Prediction Model—Reply. JAMA dermatology, 156(4), 474-474.

[8]. Vivot, A., Grégory, J., \&Porcher, R. (2020). Application of Basic Epidemiologic Principles and Electronic Health Records in a Deep Learning Prediction Model. JAMA dermatology, 156(4), 472-473.

[9]. Cho, S. I., Lee, D., \& Jo, S. J. (2020). Application of Basic Epidemiologic Principles and Electronic Health Records in a Deep Learning Prediction Model. JAMA dermatology, 156(4), 473-474.

[10]. Yue, J., Zhao, N., \& Liu, L. (2020). Prediction and Monitoring Method for Breast Cancer: A Case Study for Data from the University Hospital Centre of Coimbra. Cancer Management and Research, 12, 1887

[11]. Han, S. S., Park, I., Chang, S. E., Lim, W., Kim, M. S., Park, G. H., ...\& Na, J. I. (2020). Augmented Intelligence Dermatology: Deep Neural Networks Empower Medical Professionals in Diagnosing Skin Cancer and Predicting Treatment Options for 134 Skin Disorders. Journal of Investigative Dermatology.

[12]. Mohapatra, S., Abhishek, N. V. S., Bardhan, D., Ghosh, A. A., \&Mohanty, S. (2020). Skin Cancer Classification Using Convolution Neural Networks. In Advances in Distributed Computing and Machine Learning (pp. 433-442). Springer, Singapore.

[13]. Kravvas, G., Walker-Smith, P., Skalsky, H., Keith, D., \& Bray, A. (2020). Factors that predict complex reconstructions in facial skin cancer surgery. Dermatologic Therapy, e13933-e13933.

[14]. Huang, C. W., Nguyen, A. P., Wu, C. C., Yang, H. C., \& Li, Y. C. J. Develop a Prediction Model for Nonmelanoma Skin Cancer Using Deep Learning in EHR Data. In Soft Computing for Biomedical Applications and Related Topics (pp. 11-18). Springer, Cham. 
[15]. Matsushita, S., Nakamura, Y., Tanaka, R., Araki, R., Yamamura, K., Yoshioka, M., ...\& Nakamura, Y. (2020). Prediction of the invasive level of basal cell carcinomas in the facial area: Analysis of 718 Japanese cases. Journal of Dermatological Science.

[16]. Livieris, I., Pintelas, E., Kanavos, A., \&Pintelas, P. (2020). An improved self-labeled algorithm for cancer prediction. In GeNeDis 2018 (pp. 331-342). Springer, Cham.

[17]. Verma, A. K., Pal, S., \& Kumar, S. (2020). Prediction of skin disease using ensemble data mining techniques and feature selection method - a comparative study. Applied biochemistry and biotechnology, 190(2), 341-359.

[18]. Carroll, R. P., Ramsay, H. M., Fryer, A. A., Hawley, C. M., Nicol, D. L., \& Harden, P. N. (2003). Incidence and prediction of nonmelanoma skin cancer post-renal transplantation: a prospective study in Queensland, Australia. American journal of kidney diseases, 41(3), 676-683.

[19]. Vazquez, A. I., de los Campos, G., Klimentidis, Y. C., Rosa, G. J., Gianola, D., Yi, N., \& Allison, D. B. (2012). A comprehensive genetic approach for improving prediction of skin cancer risk in humans. Genetics, 192(4), 1493-1502.

[20]. Dwyer, T., Stankovich, J. M., Blizzard, L., FitzGerald, L. M., Dickinson, J. L., Reilly, A.,...\& Sale, M. M. (2004). Does the addition of genotype information improve prediction of the risk of melanoma and nonmelanoma skin cancer beyond that obtained from skin phenotype?. American journal of epidemiology, 159(9), 826-833.

[21]. Bhowmik, A., Repaka, R., Mulaveesala, R., \& Mishra, S. C. (2015). Suitability of frequency modulated thermal wave imaging for skin cancer detection - a theoretical prediction. Journal of thermal biology, 51, 65-82.

[22]. DrRangaSwamySirisati "Machine Learning-based diagnosis of Diabetic Retinopathy using digital Fundus images with CLAHE along FPGA Methodology \&quot;, International Journal of Advanced Science and Technology (IJAST- 2005-4238), Volume29, Issue 5 (April-2019), (pp- 12748-12759).

[23]. Dr S RangaSwamy "Dimensionality Reduction using Machine Learning and Big Data Technologies\&quot; International Journal of Innovative Technology and Exploring Engineering (IJTEE- 2278- 3075), Volume9, Issue 2 (Dec-2019), (pp- 1740-1745).

[24]. Rivas, M., Rojas, E., \&Calaf, G. M. (2012). Prediction of skin cancer occurrence by ultraviolet solar index. Oncology letters, 3(4), 893896. 